\title{
Transverse Bragg Gratings and Lasers Fabricated by Electron Beam Lithography and Reactive Ion Etching
}

Guy DeRose, Lin Zhu, and Axel Scherer

California Institute of Technology, Electrical Engineering and Applied Physics

1200 E. California Blvd, MC 200-36, Pasadena CA 91125

The development of compact and efficient laser sources at $1500 \mathrm{~nm}$ and $1300 \mathrm{~nm}$ without the need for cleaved edges has been pursued since the early 1980s with the development of vertical cavity surface emitting lasers (VCSELs) and distributed feedback lasers (DFBs). These lasers have been widely used for optical data communications, and are currently applied for high frequency interconnects. In the past, much work has been done to define higher power and higher frequency VCSELs for surface emission from InGaAsP materials. For this reason, lithographically defined photonic crystal lasers and transverse Bragg reflector (TBR) lasers have been recently developed within InGaAsP gain material emitting in the telecommunications spectrum. These geometries enabled the reduction of the mode volume to a minimum, but the resulting devices typically suffer from low gain and correspondingly small output power capability.

Recently, optimization techniques for the lithographic definition of new laser structures by directwrite electron beam lithography have been developed. Here we show promising new surface emitting DFB/DBR laser designs. To precisely define the geometries needed for efficient light sources, proximity effect correction modeling $[1,2]$ methods have also become available. After electron beam writing of the gratings in electron beam resist, complex grating structures have been etched into the InGaAsP substrate by inductively-coupled plasma / reactive ion etching (ICP/RIE). In Figure 1 we present a typical example of a cross-section through an etched TBR structure.

\section{References:}

1. W. M. J. Green, J. Scheuer, G. DeRose, and A. Yariv, "Vertically emitting annular Bragg lasers using polymer epitaxial transfer," Applied Physics Letters, 85 (2004) 3669.

2. W. M. J. Green, J. Scheuer, G. A. DeRose, A Yariv, A Scherer, "Assessment of lithographic process variation effects in InGaAsP annular Bragg resonator lasers," Journal Of Vacuum Science \& Technology B 22 (2004) 3206.

3. L. Zhu, J. M. Choi, G. A. DeRose, A. Yariv, and A. Scherer, "Electrically pumped two dimensional Bragg grating lasers," Optics Letters, in press.

The authors wish to acknowledge funding from the DARPA Center for Opto Fluidic Integration program under contract HR0011-04-1-0032. 
Figure 1. SEM micrograph of inductively coupled plasma / reactive ion etching - fabricated transverse Bragg grating.

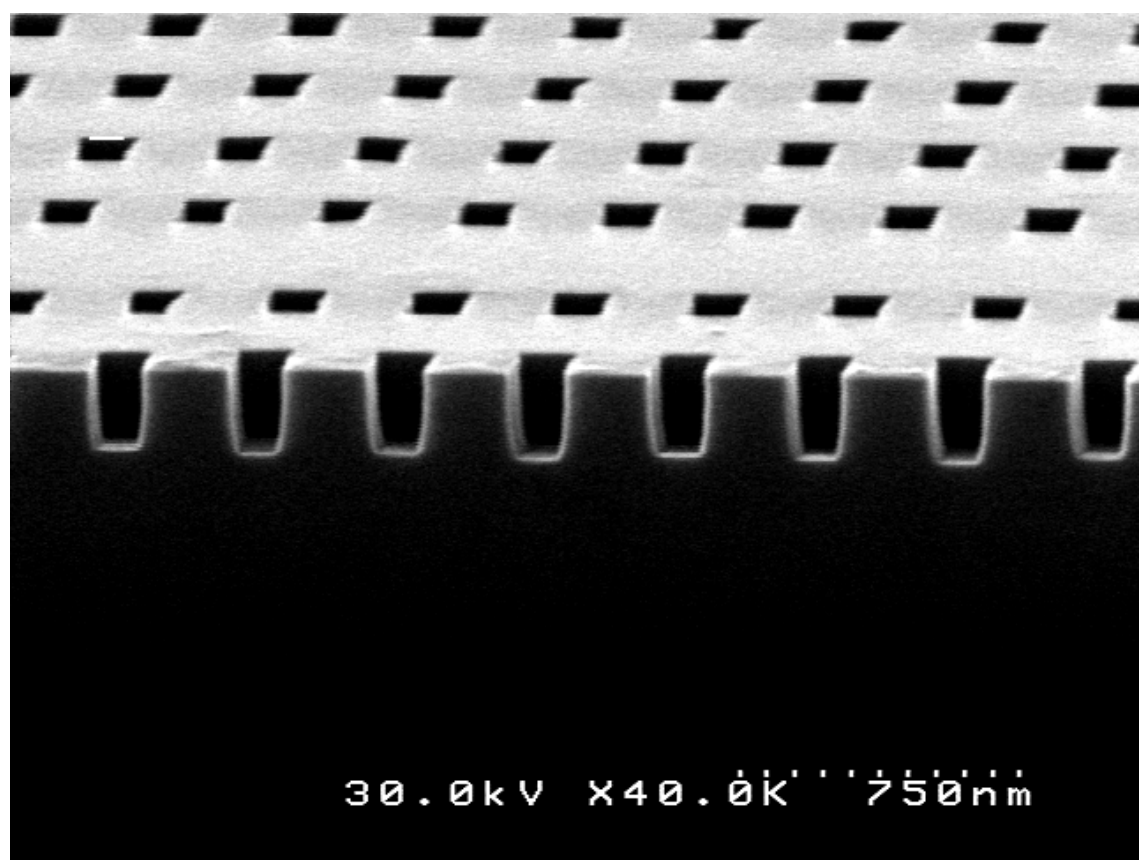

